\title{
AN ILLUMINATION INVARIANT FACE RECOGNITION USING 2D DisCRETE COSINE TRANSFORM AND CLAHE
}

\author{
A.Thamizharasi ${ }^{1}$ and Dr Jayasudha J.S ${ }^{2}$ \\ ${ }^{1}$ Research Scholar,ManonmaniamSundaranar University, Abhishekapatti, \\ Tirunelveli -627012, Tamilnadu, India \\ ${ }^{2}$ Professor,Department of Computer Science \& Engineering, \\ SCT College of Engineering,Pappanamcode, Trivandrum, Kerala, India
}

\begin{abstract}
Automatic face recognition performance is affected due to the head rotations and tilt, lighting intensity and angle, facial expressions, aging and partial occlusion of face using Hats, scarves, glasses etc.In this paper, illumination normalization of face images is done by combining $2 D$ Discrete Cosine Transform and Contrast Limited Adaptive Histogram Equalization. The proposed method selects certain percentage of DCT coefficients and rest is set to 0. Then, inverse DCT is applied which is followed by logarithm transform and CLAHE. Thesesteps create illumination invariant face image, termed as 'DCT CLAHE' image. The fisher face subspace method extracts features from 'DCT CLAHE' imageand features are matched with cosine similarity. The proposed method is tested in AR database and performance measures like recognition rate, Verification rate at $1 \%$ FAR and Equal Error Rate are computed. The experimental results shows high recognition rate in AR database.
\end{abstract}

\section{KEYWORDS}

Facerecognition, DCT CLAHE, recognition rate, AR, 2D DCT, CLAHE etc.

\section{INTRODUCTION}

Face recognition is task of identifying a person from a still image or video sequence using the faces stored in database. Face recognition is useful in security, surveillance systems, social networks, identification of faces in driving licenses etc.The methods used for solving the face recognition problem are classified as follows: Image based, Feature based and combined methods. Image based methods analyses image as an array of pixels, with shades of grey. Feature based methods analyses anthropomorphic face features, its geometry. Combined methods extract areas of features and on these areas one can design image based algorithms.

A face image has high dimension. Its dimension has to be reduced prior to face recognition [4].Turk's Eigenfacesalgorithm based on PCA is a well-known face known face representation method and it reduces the high dimensionality of face images. It provides minimal reconstruction error;it de-emphasizes high-frequency information and effectively reducing the information available for classification [1]. Principal Component Analysis (PCA) is used to reduce the dimensionality of image space [2]. Eigenfaces recognition is performed by projecting a new image into the linear subspace and then classifies the face using nearest neighbour classifier. The following section describes 2D DCT.

DOI:10.5121/ijcsit.2016.8304 


\subsection{D Discrete Cosine Transform (2D DCT)}

Discrete Cosine Transform (DCT) is a local appearance based method for face recognition. DCT possess the properties like de-correlation, Separability, energy compaction, symmetry, orthogonality etc. which makes it suitable for face recognition. The low-frequency components are compactly stored in the upper left corner. These components can be extracted for face recognition. The most common DCT definition of a 1-D sequence of length $\mathrm{N}$ is

$$
C(u)=\alpha(u) \sum_{x=0}^{N-1} f(x) \cos \left[\frac{\pi(2 x+1) u}{2 N}\right],
$$

for $\mathrm{u}=0,1,2,, N-1 \ldots$.

The inverse transformation is defined as

$$
f(x)=\sum_{u=0}^{N-1} \alpha(u) C(u) \cos \left[\frac{\pi(2 x+1) u}{2 N}\right]
$$

for $\mathrm{X}=0,1,2, \mathrm{~N}-1$. In both equations (1) and (2) $\alpha(u)$ is defined as

$$
\begin{aligned}
& \alpha(u)=\left\{\begin{array}{lll}
\sqrt{\frac{1}{N}} & \text { for } & u=0 \\
\sqrt{\frac{2}{N}} & \text { for } & u \neq 0 .
\end{array}\right. \\
& \left.C(u=0)=\sqrt{\frac{1}{N}} \sum_{x=0}^{N-1} f(x) .\right]
\end{aligned}
$$

It is clear from (1) that for $u=0$,

Thus, the first transform coefficient is referred asDC Coefficient. All other transform coefficients are called the AC Coefficient.

The 2-D DCT is a direct extension of the 1-D case and is given by

$$
C(u, v)=\alpha(u) \alpha(v) \sum_{x=0}^{N-1, N-1} \sum_{y=0} f(x, y) \cos \left[\frac{\pi(2 x+1) u}{2 N}\right] \cos \left[\frac{\pi(2 y+1) v}{2 N}\right]
$$

for $\mathrm{u}, \mathrm{v} 0,1,2,, \mathrm{~N}-1$ and $\alpha(u)$ and $\alpha(v)$ are defined in equation (3). The inverse transform is defined as

$$
f(x, y)=\sum_{u=0}^{N-1} \sum_{v=0}^{N-1} \alpha(u) \alpha(v) C(u, v) \cos \left[\frac{\pi(2 x+1) u}{2 N}\right] \cos \left[\frac{\pi(2 y+1) v}{2 N}\right],
$$

For $\mathrm{x}, \mathrm{y}=0,1,2, \mathrm{~N}-1 . \quad[5]$. 


\subsection{Contrast Limited AdAPTIVE Histogram Equalization (Clahe)}

The variants of Histogram Equalization (HE) [15] are Adaptive Histogram Equalization (AHE) and Contrast Limited Histogram Equalization (CLHE). AHE method divides the image into small contextual regions called 'tiles' and HE is applied to all the regions. The tiles are then stitched back using bilinear interpolation method. The AHE method increases the local contrast of the image but it results in noise amplification. To limit the noise amplification, CLHE method clips the height of the histogram based on threshold. Pisano et al. proposed CLAHE method for enhancing the low-contrast medical images [16]. The Contrast Limited Adaptive Histogram (CLAHE) is a combination of AHE and CLHE. CLAHE divides the image into small contextual regions called 'tiles' and does histogram clipping based on threshold. The CLAHE method enhances the local details of the image.

\section{RELATED WORKS}

The face recognition works using DCT are discussed below. Aman R et al. [7] had applied DCT to the entire image and global features are obtained. The facial features eyes, nose and mouth are also extracted and DCT is applied to these features. The weights are assigned to each feature depending upon the recognition rate obtained. The local and global features are used for comparison. The work is tested using database of 25 images. The test results of global features, local features and combining local with global shows recognition accuracy of $92.5 \%, 90.2 \%$ and $94.5 \%$ respectively.

Feature extraction using block based DCT involves dividing the image into blocks of uniform size and isolating the most relevant features of each block [8]. After DCT is applied, the DC component or low frequency components contain information useful for face recognition, while the high frequency components correspond to edges. The low frequency components are illumination sensitive and high frequency components are sensitive to pose and expression variations. Block based DCT shows recognition accuracy of $87.5 \%$ and $66.67 \%$ in ORL and Yale database respectively.

A Block-Based Discrete Cosine Transform (BBDCT) is proposed for feature extraction with $8 \mathrm{X}$ 8 DCT block size which collects the information within that block [9]. The local analysis using block based DCT is performed; the necessary features are combined for representing the extracted features. The BPSO structure based on swarm intelligence reduces the feature subset, and improves its performance [9].This method tested in ORL database using training to testing ratio of 9:1 gives recognition accuracy of 99\%. DCT with Nearest Neighbor Discriminant Analysis (NNDA) is used for face recognition [10]. Discrete Cosine Transform (DCT) extractsfacial features from face image. Since most information is present in low frequency DCT coefficients, some of them are selected and given as input for Discrimination analysis. Then using NNDA discrimination analysis is done. This method tested using training to testing ratio of 5:5 and 6:5 gives recognition accuracy of $99 \%$ and $98.5 \%$ in ORL database and Yale database respectively.

DCT is a local appearance based face representation method which is a generic local approach and it does not require detection of local regions, such as eyesin component based approaches [12]. Local appearance based face representation is performed [12]. The face image is detected and normalized. The next step is face image is divided into small blocks each of size 8x8 pixels; then each block is represented by its DCT coefficients. While representing the face image, the 
top-left DCT coefficients are removed. Then the remaining DCT coefficients containing the highest information are extracted via zig-zag scan. Using training to testing ratio of 5:6, the local DCT shows recognition accuracy of $99 \%$ in Yale database.

Xiao-Yuan Jing et al. [13] proposed an image recognition approach by combining DCT and the linear discrimination technique. It uses a two-dimensional (2-D) separability judgment for selection of useful DCT frequency bands for image classification. The linear discriminative features are then extracted by an improved Fisher face method and classified using the nearest neighbor classifier.

Weilon Chen et al. [14] had stated illumination variations mainly lie in the low-frequency band. The facial features in the dark images are recovered by applying DCT on the logarithm image. The authors adjust the brightness of the image by discarding DCT coefficients of the original image. When DCT coefficients of the logarithm image are discarded, it will adjust the illumination and recovers the face images reflectance characteristics.

\section{OBJECTIVE OF WORK}

A lot of research work for face recognition reported using DCT shows the importance of DCT in the face recognition area. Thus the proposed workdoes face recognition by normalizingthe illumination and create illumination invariant image using DCT and CLAHE. The Contrast Limited Adaptive Histogram Equalization is popular in medical images for enhancing poor contrast images. The fisher face is used for extracting the features from the illumination normalized images. Then the classification of extracted features is done usingcosine similarity. The proposed method is tested using AR database.

\subsection{ILlumination InVARIANT FACE IMAge CREATION}

The proposed method steps are as follows: The input color image is converted into gray-scale image. The gray-scale image is processed first with log transformation, and then 2D DCT is applied.Fig 1 shows the DCT coefficients arepresent as white spots in the upper left corner. A lot of research work is carried out for selection of the DCT coefficients. In this work, $25 \%, 50 \%$ and $75 \%$ DCT coefficients are selected while setting the rest to zero. Then inverse DCT is applied. Fig. 2 shows the selection of top left $25 \%$ DCT coefficients, Fig 3 shows the selection of top left $50 \%$ DCT coefficients and fig 4 shows the selection of top 75\% DCT coefficients.

After inverse DCT, the size of image is retained to original image size. Then, logarithm transform is applied and then CLAHE is applied to normalize the contrast in the image. The image obtained is termed as 'DCT CLAHE' image. The 25\%, 50\% and 75\% selected DCT coefficients are termed as DCT 25 CLAHE, DCT 50 CLAHE and DCT 75 CLAHE respectively. 
International Journal of Computer Science \& Information Technology (IJCSIT) Vol 8, No 3, June 2016

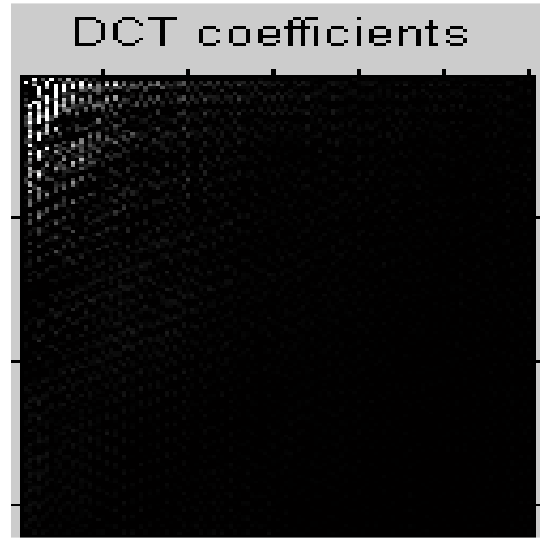

Fig 1.Dct Coefficients

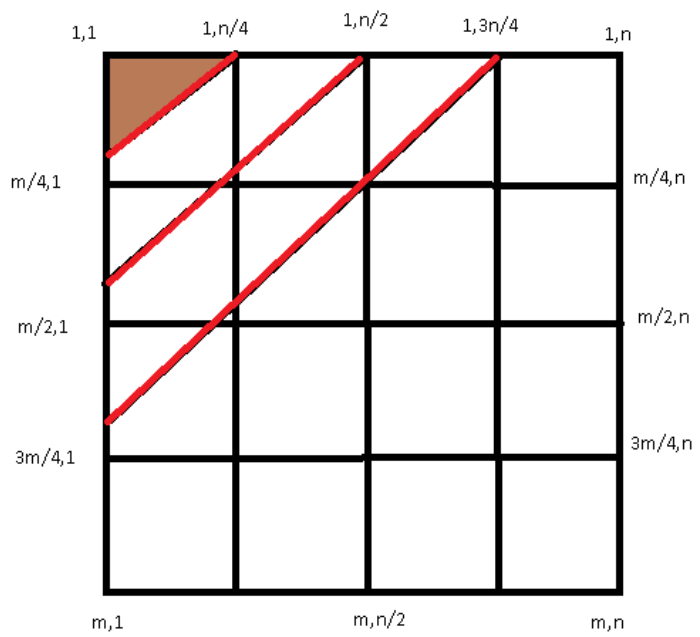

Fig.2. Selection of Dct 25 Coefficients

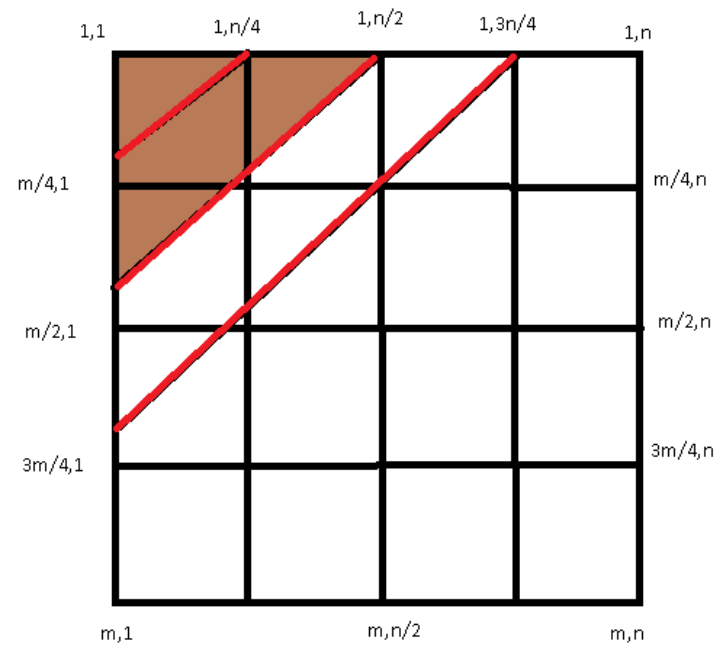

Fig. 3.Selection of Dct 50 Coefficients 


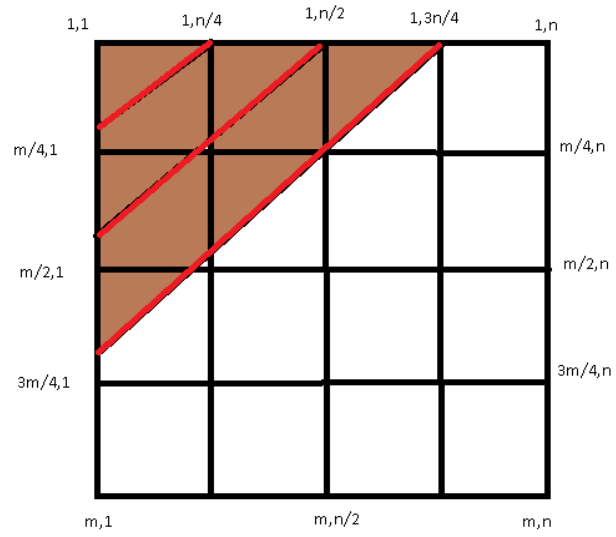

Fig. 4.Selection of Dct 75 Coefficients

The output images obtained by the illumination normalization steps are shown in fig 5 . In fig 5 , first image is original color image, second is gray image, third is the logarithm transformation image, fourth is the DCT 50 selected coefficients, fifth is the enhanced DCT 25 CLAHE image, sixth is DCT 50 CLAHE image and seventh image is DCT 75 CLAHE image. The features of enhanced DCT images is extracted using fisher face subspace projection method [11]. The featuresof test image are matched with train image using cosine similarity.

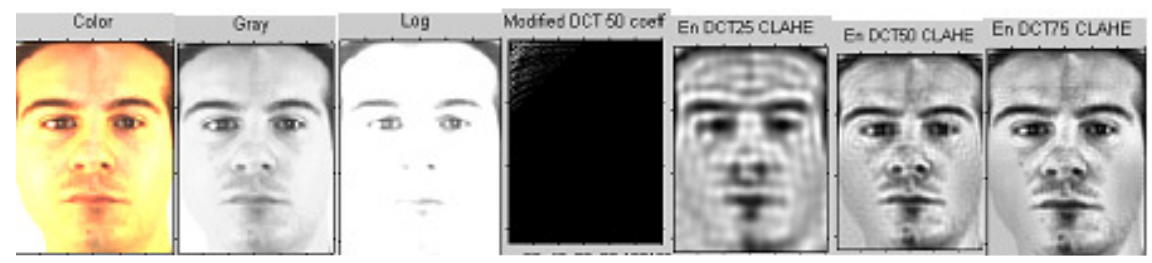

Fig.5. Output Images of the DCT CLAHE

\section{EXPERIMENTAL WORK}

The experimental work is tested using AR database. The AR database has 50 men and 50 women imageswith a total of 100 persons and images were taken in two different sessions [3]. Each image is of size $160 \times 120$ pixels. There are 26 images for each person and totally there are 2600 images. AR face image subset is created with 14 images of each person with a total of 1400 images. This subset contains images of different illuminations and different expressions. Out of the 14 images of each person, the first image in neutral condition is used as train image. The testing is done by changing the total number of train images per subject from 2 to 10 . Fig 6 shows the sample image in AR database subset.

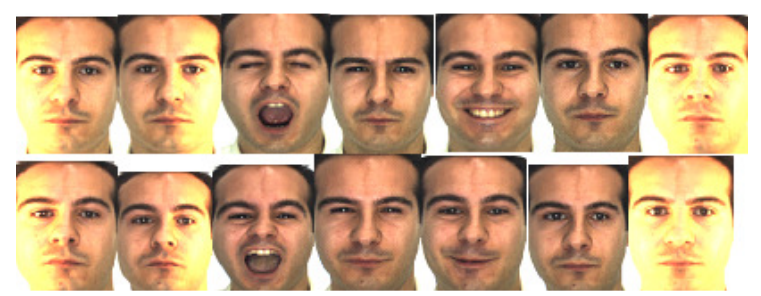

Fig.6. AR Database Sample Images in 14 Different Conditions 
There are 100 subjects in AR database. If the total number of train numbers per subject is 2, then there will be 12 images per subject for testing. Thus, train database contains 200 images and test database contains 1200 images. The testing is done on AR database by using the total number of train numbers per subject from 2 to 10 . The performance measures face recognition rate, $1 \%$ False Acceptance Rate (FAR) and Equal Error Rate (EER) are computed. The testing is repeated for DCT 25 CLAHE, DCT 50 CLAHE and DCT 75 CLAHE images. The results are shown in table 1. The results show that DCT 75 CLAHE method with six train numbers has $96.13 \%$ recognition rate and $98.25 \%$ at $1 \%$ FAR. Thus, six train numbers per subject is $43 \%$ training and $67 \%$ testing. Fig 7 shows ROC curve for DCT 75 CLAHE using fisher face cosine similarity on AR database. Fig 8 shows CMC curve for DCT 75 CLAHE using fisher face cosine similarity on AR database. The methods in literature uses block based DCT approach. However, this block based approach would result in increase in computational complexity. In the literature, the illumination sensitive component is set to zero. This method does not change the DC component as it carries useful information of the face image. The CLAHE will adjust the illumination present in the DCT. Thus this method does not suffer from computational complexity like block based methods. Thus it is a simple approach and it adjusts the illumination by preserving the face information. The results in Table.1 show that the proposed method outperforms the works discussed in related works section.

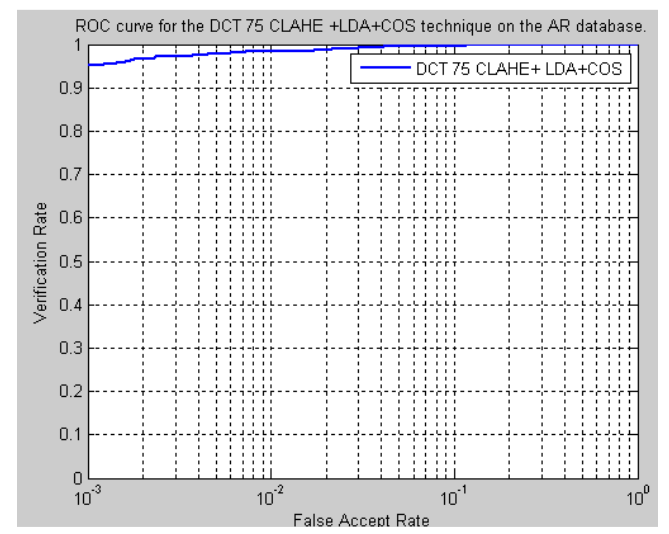

Fig 7. ROC Curve For DCT 75 CLAHE Using Fisher Face Cosine Similarity on AR Database

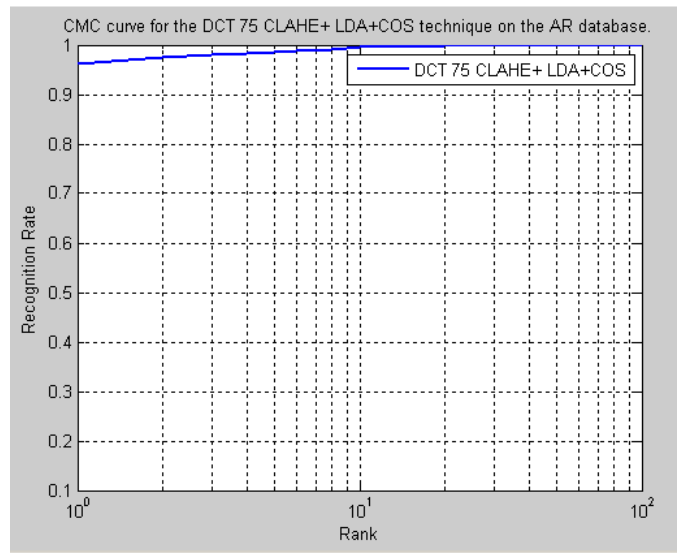

Fig 8. CMC Curve For DCT 75 CLAHE Using Fisher Face Cosine Similarity on AR Database 
Table 1.Performance Measures of the Proposed Method Using Fisher Face Feature Extraction and Cosine Similarity on AR Database

\begin{tabular}{|c|c|c|c|c|c|c|c|c|c|}
\hline \multirow[b]{3}{*}{$\begin{array}{c}\text { Performance } \\
\text { Measures in \% }\end{array}$} & \multicolumn{9}{|c|}{ DCT 25 CLAHE + Fisher face + Cosine similarity } \\
\hline & \multicolumn{9}{|c|}{ Total Number of Train Numbers per subject } \\
\hline & 2 & 3 & 4 & 5 & 6 & 7 & 8 & 9 & 10 \\
\hline Recognition rate & 81.58 & 81.82 & 82.4 & 84.89 & 89.63 & 89 & 97.83 & 98.2 & 98.75 \\
\hline $1 \%$ FAR & 86.67 & 87.82 & 87.8 & 89.44 & 94.25 & 92.57 & 98.67 & 99.4 & 99 \\
\hline EER & 5.65 & 5.57 & 6.39 & 5.68 & 3.34 & 4.01 & 1.17 & 0.8 & 1 \\
\hline \multirow{3}{*}{$\begin{array}{c}\text { Performance } \\
\text { Measures in \% }\end{array}$} & \multicolumn{9}{|c|}{ DCT 50 CLAHE + Fisher face + Cosine similarity } \\
\hline & \multicolumn{9}{|c|}{ Total Number of Train Numbers per subject } \\
\hline & 2 & 3 & 4 & 5 & 6 & 7 & 8 & 9 & 10 \\
\hline Recognition rate & 82.75 & 86.45 & 87.4 & 91.44 & 95.13 & 93.57 & 98.5 & 98.4 & 97.75 \\
\hline $1 \%$ FAR & 87.42 & 91.27 & 92.3 & 94.11 & 97.75 & 97.43 & 99.67 & 99.8 & 99.5 \\
\hline EER & 5.16 & 4.45 & 4.7 & 4 & 1.75 & 1.86 & 0.5 & 0.4 & 0.74 \\
\hline \multirow{3}{*}{$\begin{array}{c}\text { Performance } \\
\text { Measures in \% }\end{array}$} & \multicolumn{9}{|c|}{ DCT 75 CLAHE + Fisher face + Cosine similarity } \\
\hline & \multicolumn{9}{|c|}{ Total Number of Train Numbers per subject } \\
\hline & 2 & 3 & 4 & 5 & 6 & 7 & 8 & 9 & 10 \\
\hline Recognition rate & 83.08 & 86.64 & 87.6 & 91.89 & 96.13 & 94.86 & 98.67 & 99 & 98.75 \\
\hline $1 \%$ FAR & 87.58 & 91.18 & 91.7 & 94.44 & 98.25 & 98 & 99.83 & 99.6 & 99.5 \\
\hline EER & 5.25 & 4.26 & 4.7 & 3.66 & 1.5 & 1.42 & 0.33 & 0.41 & 0.5 \\
\hline
\end{tabular}

\section{CONCLUSiON}

The face recognition accuracy is made difficult by variations in illumination. Thus the proposed method does an illumination invariant face recognition using 2D DCT and CLAHE. The DCT coefficients selected are 25\%, 50\% and 75\% and DCT 25 CLAHE, DCT 50 CLAHE and DCT 75 CLAHE images are obtained respectively. Then, fisher face feature extraction is done. The cosine similarity is used for finding the matching image. The testing is done on AR database. The performance measures recognition rate, 1\% FAR and EER for all the three methods are computed. The results show that DCT 75 CLAHE has best recognition rate of $96.13 \%$ with $43 \%$ training set. However, when two train numbers per subject are used, DCT 25 CLAHE shows $81 \%$ recognition rate. The future work is to select little number of features than $25 \%$ DCT coefficients and increase the recognition accuracy.

\section{REFERENCES}

[1] Matthew A. Turk and Alex P. Pentlan, Eigenfaces for recognisation. Journal of cognitive nero sciences, Vol. 3, No. 1, 2002.

[2] L.I.Smith,A tutorial on principal component analysis, Information Fusion,Elsevier, 2002.

[3] Martinez A andBenavente R, AR Face Database, Technical Report, Computer Vision Centre, No. 24, 1998.

[4] Jamie Cook, VinodChandran, SridhaSridharan, Multiscale representation for 3-D Face recognition, IEEE Transactions on Information Forensics and Security,Vol. 2, No.3,2007.

[5] Dao-Qing Dai and Hong Yan, Wavelets and Face Recognition, in book Face Recognition, KresimirDelac and MislavGrgic (Ed.), InTech, ISBN: 978-3-902613-03-5, 2007.

[6] ZiadM.Hafed and Martin D. Levine, Face Recognition Using the Discrete Cosine Transform, International Journal of Computer Vision, Vol. 43, No.3, pp.167-188, 2001.

[7] Aman R. Chadha, Pallavi P. Vaidya, M. Mani Roja, Face Recognition Using Discrete Cosine Transform for Global and Local Features, Proceedings of International Conference on Recent 
International Journal of Computer Science \& Information Technology (IJCSIT) Vol 8, No 3, June 2016

Advancements in Electrical, Electronics and Control Engineering (IConRAEeCE), CFP1153R-ART, ISBN: 978-1-4577-2149-6, 2011.

[8] VikasMaheshkar, SushilaKamble, SuneetaAgarwal, and Vinay Kumar Srivastava, DCT Based Reduced Face For Face Recognition, International Journal of Information Technology and Knowledge Management, Vol. 5, pp. 97-100, 2012.

[9] K Manikantan, VaishnaviGovindarajan, V V S SasiKiran, S Ramachandran, Face Recognition using Block-Based DCT Feature Extraction, Journal of Advanced Computer Science and Technology, Vol.1, No.4, pp. 266-283, 2012

[10] Surya Kant Tyagi and PriteeKhanna, Face Recognition Using Discrete Cosine Transform and Nearest Neighbor Discriminant Analysis, International Journal of Engineering and Technology, Vol. 4, No. 3, pp.311-314, 2012.

[11] P.N.Belhumeur, J.P.Hespanha and D.J. Krieg man, "Eigen faces Vs. Fisher faces: Recognition using Class specific linear projection”, IEEE Trans. Pattern Analysis and Machine Intelligence, Vol. 19, No. 7, pp. 711-720, 1997.

[12] Hazim Kemal Ekenel, Rainer Stiefelhagen, Local Appearance Based Face Recognition using Discrete Cosine Transform, 13th European Signal Processing Conference (EUSIPCO), 2005.

[13] Xiao-Yuan Jing and David Zhang, A Face and Palm print Recognition Approach Based on Discriminant DCT Feature Extraction, IEEE Transactions on Systems, Man and Cybernetics, Vol.34, No.6, pp.2405-2415, 2004.

[14] Weilong Chen, MengJooEr, and Shiqian Wu, Illumination Compensation and Normalization for Robust Face Recognition Using Discrete Cosine Transform in Logarithm Domain, IEEE Transactions on Systems, Man and Cybernetics, Vol. 36, No. 2, 2006

[15] Rafael C. Gonzalez and Richard E. Woods, Digital Image Processing, Addison- Wesley, 1993.

[16] E. D. Pisano, S. Zong, B. M. Hemminger, M. DeLuca, R. E. Johnston, K. Muller, M. P. Braeuning and S. M. Pizer, Contrast Limited Adaptive Histogram Equalization Image Processing to Improve the Detection of Simulated Spiculations in Dense Mammograms, Journal of Digit Imaging, Vol. 11, No. 4, pp. 193-200, 1998.

[17] Martinez A andBenavente R, AR Face Database, Technical Report, Computer Vision Centre, No. 24, 1998. 